\title{
REAL-WORLD EVIDENCE OF DENGUE BURDEN ON HOSPITALS IN MEXICO: INSIGHTS FROM THE AUTOMATED SUBSYSTEM OF HOSPITAL DISCHARGES (SAEH) DATABASE
}

\author{
Alejandro E. Macías ${ }^{1 *}$, Esteban Puentes-Rosas ${ }^{2}$, David Velandia ${ }^{3}$, David Morley ${ }^{4}$, \\ LAURENT COUDEVILLE ${ }^{3}$ AND NiCOLAS BAURIN ${ }^{3}$ \\ ${ }^{1}$ Microbiology Area, Department of Medicine, Universidad de Guanajuato, Guanajuato, Mexico; ${ }^{2}$ Sanofi Pasteur, \\ Mexico City, Mexico; ${ }^{3}$ Sanofi Pasteur, Lyon, France; ${ }^{4}$ Ariana Pharmaceuticals, Paris, France.
}

\begin{abstract}
Background: The incidence of dengue in Mexico has increased in recent decades. It has been suggested that dengue outbreaks may compromise treatment quality in hospitals. Objective: The objective of the study was to quantify the burden imposed by dengue on hospital services in Mexico. Methods: We analyzed 19.2 million records contained in the database of hospital services of the Mexican Ministry of Health between 2008 and 2014. The number of admissions due to dengue was compared to other potentially preventable hospitalizations. Hospital departments were categorized to reflect denguerelated activity as high dengue activity (HDA), low dengue activity (LDA), or zero dengue activity departments, and the impact of dengue activity on general in-hospital mortality in HDA departments was assessed. Results: Dengue was the cause of more hospital admissions than most of the potentially preventable prevalent acute and chronic conditions and other infectious diseases. In HDA departments, dengue patient load was found to be a significant risk factor for overall in-hospital mortality. There was an approximately two-fold higher dengue case-fatality rate in LDA versus HDA departments, irrespective of dengue severity. Conclusions: This study confirms that dengue is an important cause of hospitalization in Mexico and highlights the impact of dengue activity not only on dengue case-fatality rate but also on the overall in-hospital mortality. (REV INVEST CLIN. 2019;71:168-77)
\end{abstract}

Key words: Dengue. Burden. Mexico. Hospitalization.

Corresponding author:

*Alejandro E. Macías

Microbiology Area, Department of Medicine

Universidad de Guanajuato

Guanajuato, Mexico

E-mail: aaeemmhh@yahoo.com

Received for publication: 20-07-2018

Approved for publication: 07-11-2018

DOI: $10.24875 / R I C .18002681$ 


\section{INTRODUCTION}

Dengue, a vector-borne viral disease caused by four serotypes (dengue virus [DENV]-1-4) and transmitted by Aedes aegypti mosquitoes ${ }^{1}$, is endemic in most Latin American countries, and an important cause of hospitalization and death among children and adults in the region ${ }^{2}$. All four virus serotypes are capable of causing illness and are known to circulate in the region, with cocirculation of two or more serotypes at any given time in some countries. Such cocirculation of several serotypes has the potential to cause severe disease outbreaks ${ }^{3}$.

In the Americas, 10.6 million dengue cases and 6542 deaths caused by dengue were reported to the PanAmerican Health Organization (PAHO)/World Health Organization (WHO) between 2008 and 20144. Of these, 1,025,825 dengue cases and 653 dengueassociated deaths were reported in Mexico. Annually, Mexico has accounted for $2.8-21.7 \%$ of reported dengue cases and $1.7-21.1 \%$ of dengue-associated deaths in the Americas. Case fatality rates (CFR) have ranged in Mexico from $0.04 \%$ to $0.10 \%{ }^{4}$. Between 2008 and 2014, the number of dengue cases reported in Mexico annually had ranged from 25,040 to 249,886 . Although the PAHO data are comprehensive, there is usually significant underreporting inherent in surveillance systems, which hinders assessment of the true burden of the disease. The level of under-reporting of dengue cases in Mexico at the national level may be as high as 46 -fold 5 . Indeed, dengue seroprevalence was reported to be about $75 \%$ in typical endemic communities in Mexico ${ }^{6,7}$, suggestive of high dengue transmission rates in the country even in areas considered of low dengue transmission based on historical epidemiological surveillance data.

Although there are numerous reports on the medical and economic burden of dengue, the impact of the disease during outbreaks in terms of health-care management outcome of other morbid conditions unrelated to dengue remains to be adequately explored. Since dengue outbreaks are usually clustered in time or location - cases have been shown to be clustered mainly in two coastal areas of Mexico, the Pacific and Gulf $^{8}$ - they may place a considerable burden on health-care systems and compromise treatment quality and decisions across all treatment wards, including degrading performance of clinical laboratories in the affected regions ${ }^{9}$. The information (clinical, financial, and operational) collated in hospital databases can provide insights into resource utilization ${ }^{10}$ and could be used to help determine the impact of dengue on health-care demand and other morbid conditions at a given time and location.

The Mexican public health-care system is divided into two groups. One group comprises social security institutions such as the Mexican Social Security Institute (IMSS), Institute of Social Security and Services of State Workers (ISSSTE), Mexican Oil Company (Petróleos Mexicanos, PEMEX), and the Armed Forces (SEDENA and SEMAR), and covers workers in the formal sector of the economy. The other group comprises institutions that cover the population without social security/insurance plans such as the Ministry of Health (SSa), the State Health Services (SESA), and the IMSS-Opportunities Program ${ }^{11}$. Here, we used hospital admission records in the database of hospital services (Automated Subsystem of Hospital Discharges; SAEH) from the Ministry of Health in Mexico, which typically cover public institutions for those without social security/insurance plans ${ }^{11}$, to explore the extent of the burden imposed by dengue on hospital services in the country. Specifically, we compared dengue-associated hospital admissions with other potentially preventable hospitalizations ( $\mathrm{PPHs})^{12}$, assessed the impact of dengue activity on in-hospital mortality from other morbid conditions, and explored the link between dengue activity and dengue-related mortality.

\section{METHODS}

\section{Data sources}

SAEH contains anonymized information on cases regarding reasons for hospitalization, diagnostic and treatment procedures, patient outcome, reasons for discharge, and duration of hospital stay for all causes, grouped according to the International Statistical Classification of Diseases and Related Health Problems version $10(\mathrm{ICD}-10)^{13}$. For the period assessed (2008-2014), the SAEH database included data on 19.2 million hospital admission records from 3370 departments in 817 hospitals. The Ministry of Health 
hospitals provided $38.3 \%$ of the hospital services in the country ${ }^{14}$.

Dengue-related hospitalizations were identified using ICD codes for dengue cases: A90 (dengue) or A91 (dengue hemorrhagic fever). The selection of other $\mathrm{PPH}$ for comparison to dengue was based on those proposed by the Australian Institute of Health and Welfare ${ }^{12}$. ICD codes of conditions selected are shown in supplementary information Table S1. The Australian codes were chosen as these are the most referenced in the literature, are updated annually to ensure reliability, and include vaccine PPHs (VPPHs) (Table S1) ${ }^{15}$.

The duration of hospital stay documented for each admission was used to build daily tables, per hospital departments, with calculated metrics as follows: (1) the daily dengue patient load in the hospital department was defined as the number of beds attributed to dengue according to the ICD10 classification on admission; (2) the bed capacity on any given day, calculated as the maximum number of daily beds occupied over the past 180 days in a given hospital department; and ( 3 ) the relative daily dengue patient load calculated as the ratio of the former two metrics, i.e., the percentage of beds attributed to dengue on any given day with respect to the estimated bed capacity of the hospital department.

There were 613 small departments that were unclassified (and excluded from further analysis) as they recorded very low $(<200)$ total admissions over the period 2009-2014. Hospital departments were categorized into high dengue activity (HDA), low dengue activity (LDA), or zero dengue activity (ZDA) based on dengue-related hospital admission data from the years 2009 to 2014. HDA departments were defined as those with at least 200 dengue admissions and a maximum relative daily dengue patient load exceeding $20 \%$ of the estimated bed capacity of the department. ZDA departments were those with no recorded dengue admissions. The LDA departments were by definition the remaining departments (neither HDA nor ZDA) and included those hospital departments with $<200$ total dengue admissions (but with at least one dengue admission) or a maximum relative daily dengue patient load $<20 \%$ of the estimated bed capacity of the department over 2009-2014.

\section{Outcome measures}

The main outcome measures were dengue hospital admissions (where dengue was considered the principal or secondary diagnosis) in the SAEH hospital database from 2008 to 2014, by age group, severity (ICD codes for admission were A90 [dengue] or A91 [dengue hemorrhagic fever]), whether a death occurred, and by category of hospital department (HDA, LDA). Dengue-related hospital admissions (i.e., where dengue was considered the principal diagnosis) were compared to admissions due to other PPHs or chronic conditions. The impact of dengue on general (i.e., non-dengue) in-hospital mortality of a hospitalized episode was explored using the dengue patient load (see definition below).

\section{Statistical analysis}

Analytics platforms used were MySQL Workbench 6.3.6 for storage ${ }^{16}$, and KNIME 3.4.2 and R 3.3.2 ${ }^{17}$ for analysis of the data. An ordinary logistic regression model ${ }^{18}$ was generated based on all documented admissions to HDA departments from 2009 to 2014 (553,000 episodes) and used to quantify if department dengue patient load was a risk factor for inhospital mortality. The model was developed to take into account possible confounding effects: age group, principal diagnosis defined according to the ICD $10^{13}$, department characteristics (size, specialty, and intensive care unit), and seasonality (admission year and quarter). The dengue patient load and department size factors assigned to each hospitalized episode were the average values during the episode determined from the admission and discharge dates.

\section{RESULTS}

\section{Hospital department data}

Overall, 68,000 hospitalized dengue admissions, where the disease was considered the principal or secondary diagnosis (A90 and A91), were included in the current analysis. There were 79 HDA departments, managing $55 \%$ of dengue admissions, 923 LDA departments managing $45 \%$ of dengue admissions, and 1755 ZDA departments from 2009 to 2014 (Fig. 1). 
Figure 1. Level of dengue activity recorded in the three types of hospital departments (high dengue activity, low dengue activity, and zero dengue activity) from 2009 to 2014 in Mexico. Data based on admissions where dengue was considered the principal or secondary diagnosis (WHO's ICD codes A90 and A91).

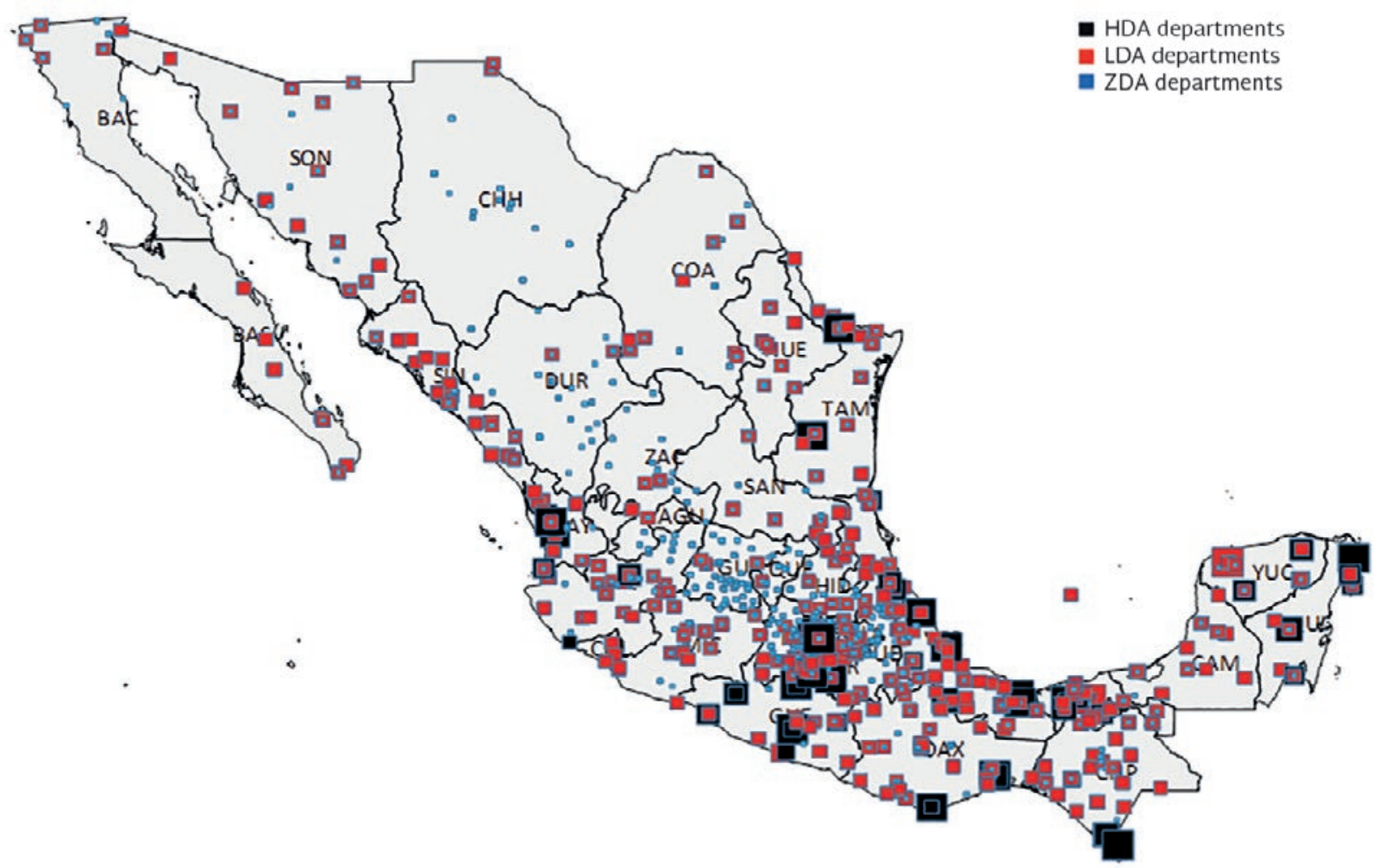

HDA: high dengue activity; LDA: low dengue activity; ZDA: zero dengue activity.

Figure 2. Weekly dengue admissions in the Mexican SAEH hospital database for the study period 2008-2014; data shown for admissions where dengue was considered the principal diagnosis (A90 and A91).

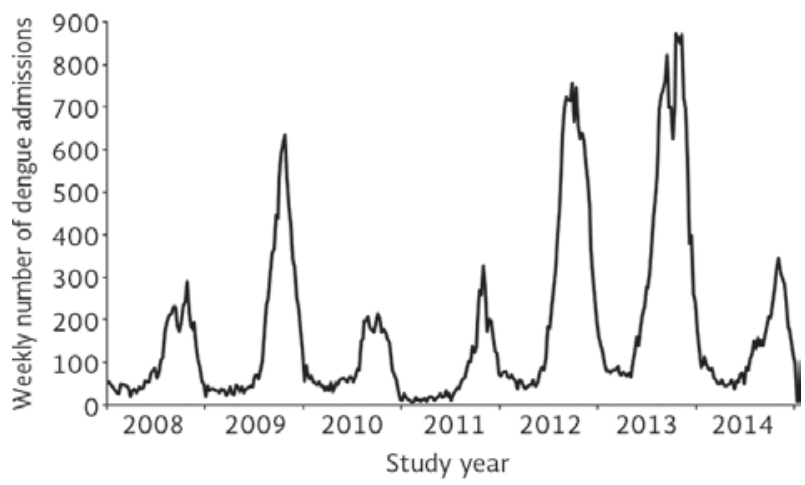

\section{Dengue activity compared to other PPHs}

Weekly dengue admissions where the disease was considered the principal diagnosis (A90 and A91) in the SAEH hospital database are shown in figure 2. There was a clear peak in dengue activity in the last quarter of each calendar year. The last quarters of 2009, 2012, and 2013 had the largest peak in weekly dengue admissions in the SAEH hospital database. Results from the SAEH database from 2008 to 2014 suggested that dengue consumed more hospital resources than other VPPHs (Fig. 3a). In 2013, dengue represented $87 \%$ of VPPHs (Fig. 3b-i; ranked 1 vs. other VPPHs), and dengue patients utilized more hospital beds than prevalent chronic conditions (ranked 2 vs. chronic conditions) such as chronic obstructive pulmonary disease, hypertension, or asthma, although not as many as diabetes (Fig. 3b-ii). Dengue patients occupied as many beds as prevalent acute conditions such as urinary tract infections (Fig. 3b-iii; ranked joint first with urinary tract infections), and dengue patients occupied more hospital resources than any other infectious disease (ranked 2 vs. other infectious diseases), except for intestinal diseases (Fig. 3b-iv). 
Figure 3. (a) Proportion of admissions (as a proxy of hospital resource utilization) attributed to potentially vaccine-preventable hospitalizations (VPPHs), for the study period 2008-2014, in Mexico. (b i-iv) A series of pie charts illustrating the proportion of dengue hospital admissions compared to a range of other conditions, in 2013, which was the peak dengue activity year within this study; (i) represents the dengue hospital admissions as a proportion of VPPHs; (ii) dengue hospital admissions as a proportion of prevalent chronic conditions; (iii) dengue hospital admissions as a proportion of prevalent acute conditions; and (iv) dengue hospital admissions as a proportion of infectious diseases. Dengue data presented for admissions where the disease was considered the principal diagnosis (A90 and A91).

A $2008-2014$

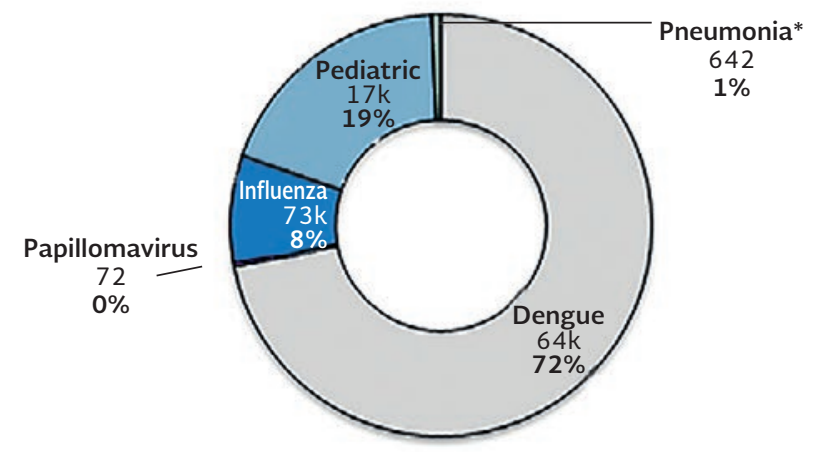

2013

i. VPPH

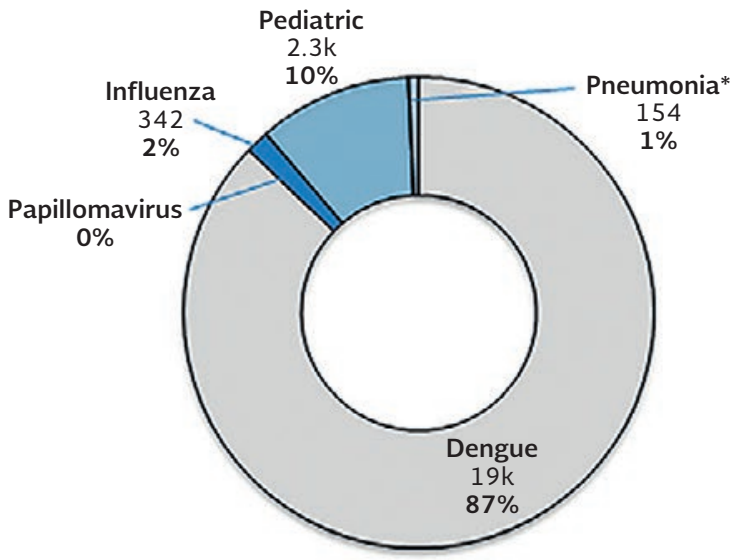

iii. Acute conditions

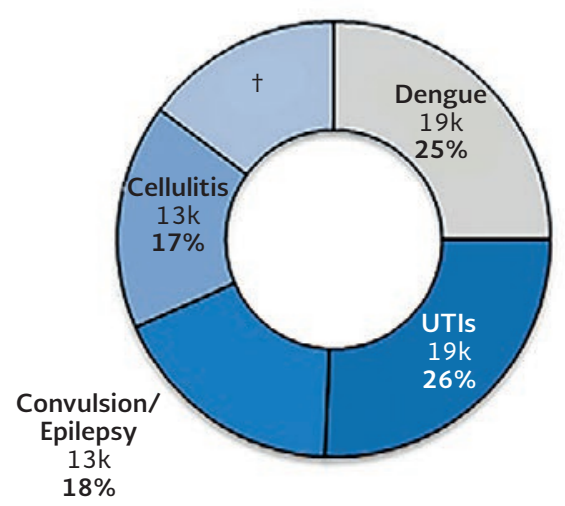

ii. Chronic conditions

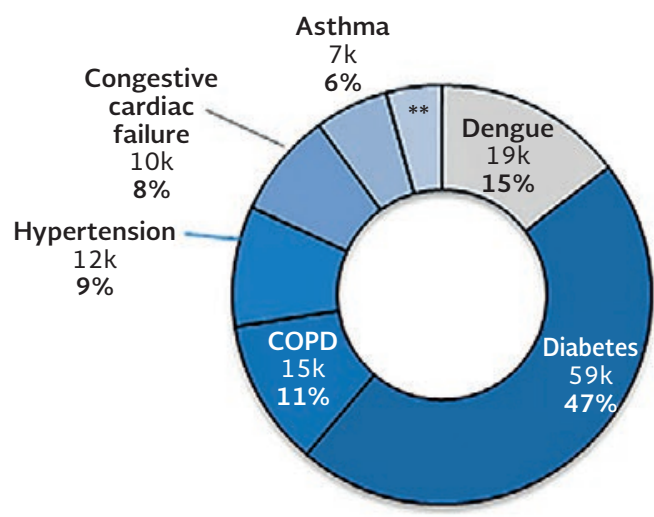

iv. Infectious diseases

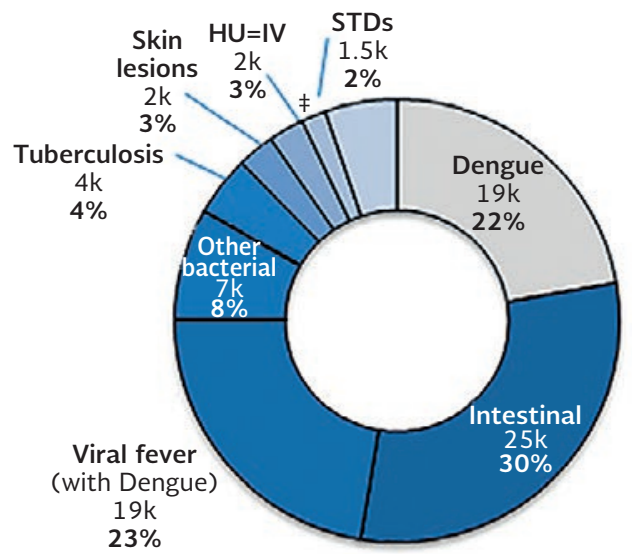

VPPH: Vaccine Potentially Preventable Hospitalization; Pediatric: Diphtheria, Haemophilus, Hepatitis B, Measles, Mumps, Pertussis, Polio, Rotavirus, Rubella Tetanus, Varicella; COPD: Chronic obstructive pulmonary disease; UTIs: urinary tract infections; HIV: human immunodeficiency virus; STDs: Sexually transmitted diseases; *Pneumonia due to Streptococcus pneumonia; "Other conditions: angina; nutritional deficiencies; anaemia; rheumatic heart diseases; bronchiectasis; ${ }^{\dagger}$ Others conditions: dental; pelvic inflammation, eclampsia, bleeding ulcer, ear, nose, and throat infections; gangrene; pneumonia; ${ }^{\ddagger}$ Other conditions: viral hepatitis; helminthiases; mycoses; viral prions; otherviral diseases; zoonotic bacterial; protozoan diseases; rickettsioses; sequelae of infections; infestations; chlamydiae; other bacterial and viral infections; other spirochetal diseases; other human herpes virus. 
Table 1. Impact of dengue patient load on in-hospital mortality odds for all conditions in HDA departments.

\begin{tabular}{ccccc}
\hline $\begin{array}{c}\text { Dengue patient } \\
\text { load range }\end{array}$ & $\begin{array}{c}\text { Hospitalized } \\
\text { episodes }\end{array}$ & $\begin{array}{c}\text { Mortality } \\
\text { odds ratio }\end{array}$ & $95 \% \mathrm{Cl}$ & Significance \\
\hline 0 & 318,321 & 1.000 & $(0.992-1.061)$ & $\mathrm{ns}$ \\
0.5 & 51,898 & 1.026 & $(1.024-1.101)$ & $* *$ \\
1 & 50,758 & 1.062 & $(1.083-1.160)$ & $* * *$ \\
$3.5-56$ & 61,961 & 1.120 & $(1.200-1.291)$ & $* * *$ \\
\hline
\end{tabular}

${ }^{* * *} p<0.001 ;{ }^{* *} p<0.01 ;{ }^{*} p<0.05$; ns: non-significant $p>0.05$; Cl: confidence interval, HDA: high dengue activity.

Dengue patient load: number of beds occupied by dengue patients (average values of the daily dengue patient load over the duration of the hospitalized dengue episode determined from the admission and discharge dates)

\section{In-hospital mortality related to dengue patient load}

Dengue daily load was shown to be a significant risk factor for overall in-hospital mortality (i.e., non-dengue-related mortality) in HDA departments. Multivariate logistic regression analysis indicated that high dengue patient load, older patient age, and larger department size increased the odds of in-hospital mortality in HDA departments (Table S2). After controlling for possible confounding factors, the odds of in-hospital death increased significantly and monotonically with increasing dengue patient load (Table 1). Mortality odds increased by $24.5 \%$ (20.0-29.1\%, $p<0.001$ ) for dengue patient load $>3$.

\section{Dengue CFR related to hospital department category}

Figure 4 shows the dengue CFR rates stratified by age, HDA and LDA departments, and according to the dengue severity (A90, A91). Higher dengue CFRs were observed in LDA than HDA departments irrespective of dengue severity. For the study period, 2009-2014, the average A90 CFRs were $0.35 \%$ for LDA and $0.18 \%$ for HDA hospital departments, and the average A91 CFRs were $1.14 \%$ and $0.55 \%$, respectively. For all ages, there was an approximately two-fold higher dengue CFR in LDA than HDA hospital departments; this difference was greater in the $\geq 60$ years of age population irrespective of dengue severity.

\section{DISCUSSION}

This study examined a large data set derived from a total of 3370 hospital departments (in 817 hospitals) across Mexico. A total of 68,000 hospitalized dengue cases were included in the analysis as well as the remaining 19.2 million admissions for comparison. Weekly data of dengue hospital admissions analyzed from the Mexican SAEH database showed peaks in dengue activity in the second half of each calendar year between 2008 and 2014. This seasonal pattern of dengue disease, with most cases occurring in the second half of the year, has previously been reported in Mexico ${ }^{19}$. The yearly variation in dengue incidence may be related to seasonal fluctuations in climate parameters influencing dengue transmission cycles; for example, an increase in sea-surface temperature, minimum temperature, and rainfall was shown to be associated with increased reported cases of dengue in the coastal municipalities of the Gulf of Mexico ${ }^{20}$. However, despite the seasonal pattern, our study showed that a number of dengue cases continued to be reported throughout the year.

As dengue admission comprised a small proportion of all hospitalizations, we chose to initially compare dengue with other preventable diseases. We found that dengue accounted for the majority of VPPHs in our study; $72 \%$ of VPPHs during our study period were due to dengue, which increased to $82 \%$ in 2013, the year with the largest peak in weekly dengue admissions. Moreover, dengue admissions were usually ranked either first or second most frequent cause of PPHs as a proportion of acute conditions, chronic conditions, and infectious diseases. These results show that dengue imposes a significant burden on hospital resources in Mexico and reflects the positive impact that the national immunization strategy has had on the public health of Mexico 21 . 
Figure 4. Dengue case fatality rates in high dengue activity and low dengue activity departments in Mexico according to the disease severity (A90 or A91). Data presented for admissions where dengue was considered the principal or secondary diagnosis.

A

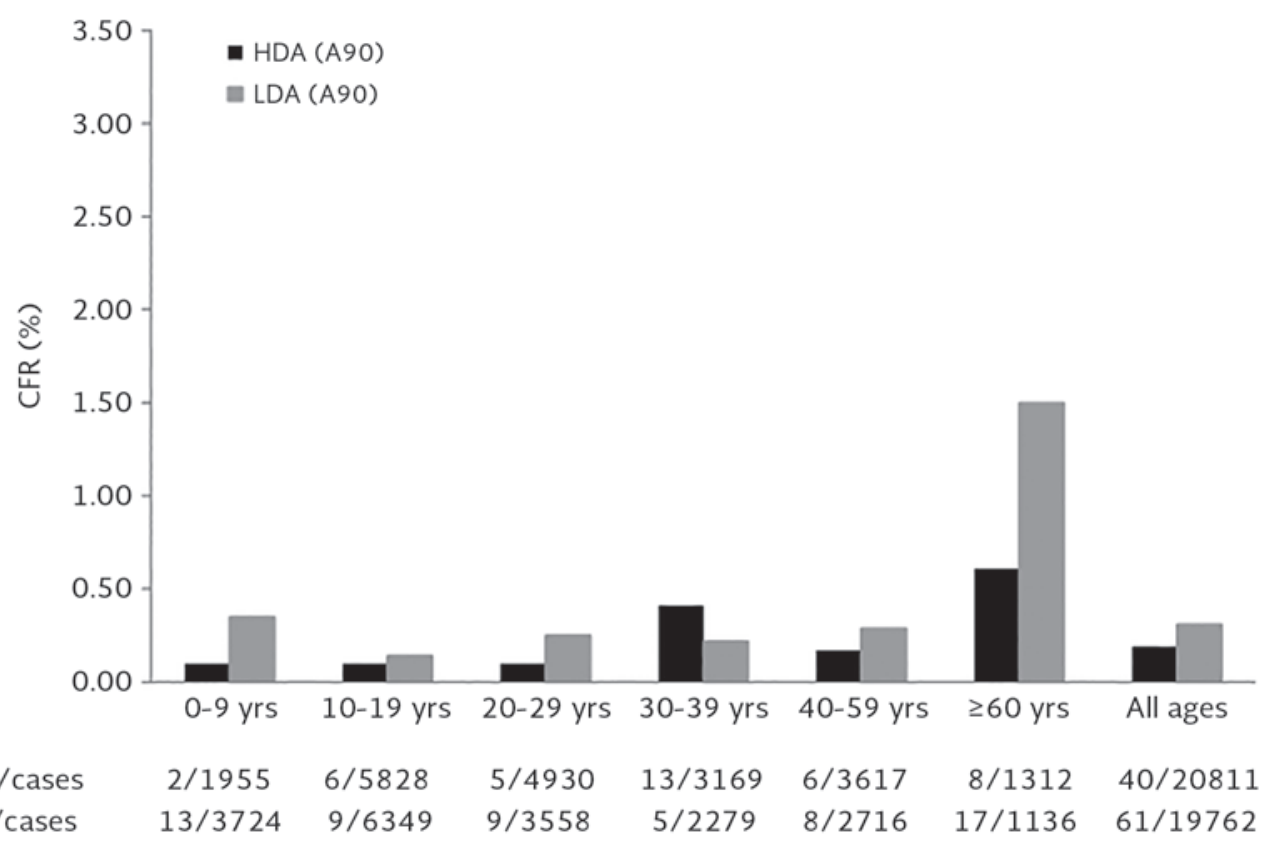

B

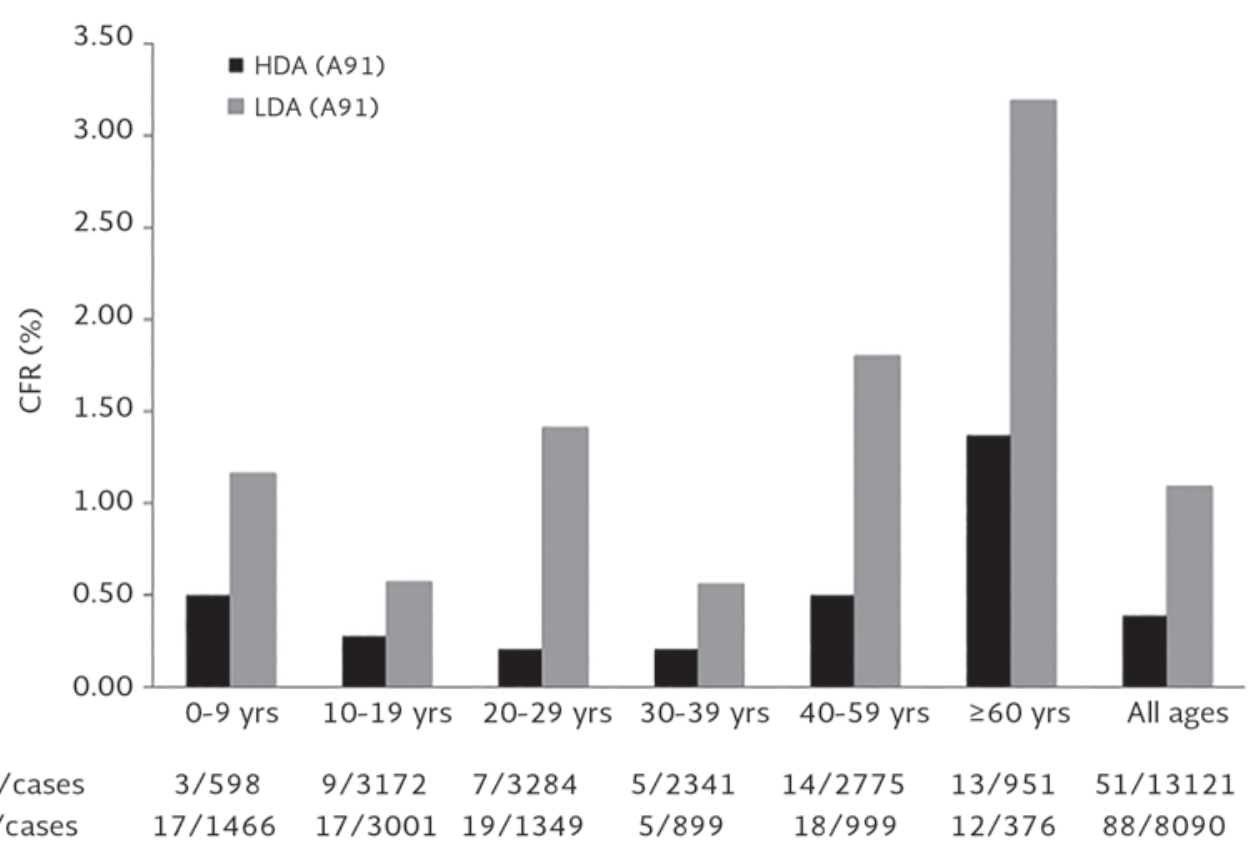

CFR: case fatility rate; HDA: high dengue activity hospital departament; LDA: low dengue activity hospital department; A90: dengue; A91: dengue hemorrhagic fever.

In dengue epidemic years, the burden may be severe enough to have an adverse impact on the health-care system's performance, as physicians dedicate less time to their patients, and demand increases for laboratory resources ${ }^{22}$, which would also indirectly affect the quality of care for other illnesses/conditions and their outcomes. Indeed, a recent study suggested that dengue outbreaks had the potential to 
undermine medical, social, economic, and political sectors in the wider society ${ }^{23}$. Such outbreaks are generally characterized by a rapid patient influx into the health-care system which may quickly become overwhelmed, as well as placing a financial burden on governments and households that could lead to wider political and social disruption and disengagement on outbreak control measures. Other potential negative results of dengue outbreaks, as seen in other epidemics, are the reductions in admissions related with nonurgent procedures and the reduction in the availability of general hospital resources 24,25 . The seasonal nature of dengue may also have an impact on the management of dengue hospitalization; it has previously been observed that a larger proportion of cases was managed in LDA departments during dengue epidemic periods ${ }^{26}$. In our study, since the CFR for dengue patients was higher in LDA departments, the fact that these departments receive more patients during outbreaks implies a higher mortality risk for those dengue patients they treat. We also confirm the disruption due to dengue on the quality of care as a whole, with dengue patient load being a significant risk factor for overall non-dengue-related inhospital mortality in HDA departments; the odds of in-hospital death increased by $24.5 \%$ for dengue patient loads $>3$. We hypothesize that the worse prognosis for patients in LDA departments is related to the lack of experience in the management of severe or evolving dengue disease. To improve the prognosis, a training program for the management of dengue should be mandatory as well as compliance with management guidelines.

We also observed dengue-related CFRs for severe cases (A91) of $0.39 \%$ and $1.09 \%$ in HDA and LDA settings, respectively, which are higher than those reported for Mexico (0.02-0.32\% CFR) published by PAHO (official figures were based on severe cases reported by all the health units) ${ }^{27}$. Although there have been important variations in the dengue mortality rates in Mexico over the last couple of decades, the mortality by age group has previously been shown to be mainly toward the extremes of life, with high mortality rates in that aged $<1$ year and $\geq 60$ years $^{28}$. Moreover, dengue mortality has consistently been highest in those aged $\geq 60$ years in the three decades to 2009 . Our data confirm the high mortality rate in those aged $\geq 60$ years and was irrespective of disease severity reported. The higher mortality rate in those aged $\geq 60$ years may be related to the more atypical disease presentation which can delay diagnosis and lifesaving interventions, combined with the higher likelihood of underlying comorbidities ${ }^{29}$. The high prevalence of comorbidities in Mexico must be highlighted. Recent models have proposed that, for 2020, $26.3 \%$ of the population 50 years and older will have been medically diagnosed with diabetes, for instance ${ }^{30}$.

We observed for all age groups an approximately two-fold higher dengue CFR in LDA than HDA hospital departments; this difference was more pronounced among those aged $\geq 60$ years irrespective of dengue severity (A90 and A91 dengue admissions). Denguerelated CFRs were also higher in LDA than in HDA departments in another similar study undertaken in Brazil $^{26}$. There are a number of reasons why denguerelated CFRs may be higher in LDA departments. These hospital departments may have less expertise/ experience in the care of patients with dengue. There is also evidence to suggest that hospitalized dengue patients treated by dedicated medical teams experienced in the clinical management of dengue would substantially improve dengue-related quality of care and clinical outcome ${ }^{31}$. Other reasons may include that LDA departments might be less prepared or equipped to provide adequate diagnostic and supportive care to dengue patients ${ }^{32}$, or lack clinical experience and adequately trained staff in the management of dengue symptoms ${ }^{31,33}$.

Overall, these results support the case for preventative measures (e.g., vector control, disease awareness/protection, and dengue vaccine) in limiting the extent of any potential dengue outbreak. However, the use of the dengue vaccine appears to confer protection among dengue-seropositive individuals but predisposes those dengue-seronegative to severe dengue ${ }^{34}$. As such, the WHO Strategic Advisory Group of Experts on immunization preferred approach to dengue vaccination in endemic settings is to screen for prior dengue infection, to ensure that only those with previous dengue infection are vaccinated ${ }^{35}$.

To the best of our knowledge, this is the largest retrospective analysis conducted in Mexico exploring dengue disease and its potential influence on hospital admissions and non-dengue patient mortality. We used the SAEH database over 7 years of continuous 
hospital activity, which represents a robust data set over a substantial time period. In addition, the quality of records examined was good; $99 \%$ of the admissions analyzed had complete records. The use of "real-world evidence" has enabled us to further characterize the true burden of dengue on hospital practice as well as on direct and indirect dengue-related mortality. However, there were some limitations to this study; the databases used captured only mortality in public hospitals run by the Mexican Ministry of Health, and so these findings may not necessarily be applicable across private hospitals or to the broader Latin American population. We used dengue admissions and mortality as indicators of disease burden, although most of the burden is associated with nonhospitalized and nonfatal cases ${ }^{9,36}$. In addition, dengue diagnosis may be challenging as a number of disease-causing pathogens (such as Zika or Chikungunya) may cause symptoms similar to dengue that are difficult to differentiate in the absence of laboratory confirmation ${ }^{37}$. The logistic regression did not account for clustering effects at the hospital or regional levels, which could lead to more accurate comparisons of the potential drivers of overall mortality at a fine geographical level. There also remain gaps in the epidemiological knowledge with regard to local serotype distribution in Mexico ${ }^{38}$.

In summary, our study confirms the important burden due to dengue in Mexican hospitals when compared to other preventable chronic and acute hospitalizations, as well as preventable hospitalizations due to other infectious diseases. The study also highlights the impact of dengue activity not only on the dengue case-fatality rate but also on the overall in-hospital mortality. It is hoped these data will help inform policy regarding resource allocation to improve the quality of care and clinical outcome of all hospitalized patients during periods of high dengue activity.

\section{ACKNOWLEDGMENTS}

This study was funded by Sanofi Pasteur, including the service provision of database consolidation, data mining and analysis by Ariana Pharmaceuticals. Editorial assistance with the preparation of the manuscript was provided by Rebecca Hornby, inScience Communications, Springer Healthcare, Chester, UK, funded by Sanofi Pasteur. The authors also thank Jean-Sébastien Persico for editorial assistance and manuscript coordination on behalf of Sanofi-Pasteur.

\section{SUPPLEMENTARY DATA}

Supplementary data are available at Revista de Investigación Clínica online (www.clinicalandtranslationalinvestigation.com). These data are provided by the corresponding author and published online for the benefit of the reader. The contents of supplementary data are the sole responsibility of the authors.

\section{CONFLICTS OF INTEREST}

AEM received travel expenses and honoraria to participate in meetings with Sanofi Pasteur. DM is an employee of Ariana Pharma. DV worked as an intern at Sanofi Pasteur in Lyon. All other authors are employees of Sanofi Pasteur.

A preliminary version of these results was presented at the SLIPE (Latin American Society of Pediatric Infectious Diseases) Congress held in Cancun, Mexico in November 2017.

\section{REFERENCES}

1. Ebi KL, Nealon J. Dengue in a changing climate. Environ Res. 2016;151:115-23.

2. WHO. World Health Organization, Dengue and Severe DengueFact Sheet; 2017. Available from: http://www.who.int/mediacentre/factsheets/fs117/en/. [Last Accessed 2017 Dec 1].

3. Nogueira RM, Miagostovich MP, Schatzmayr HG, et al. Dengue in the state of Rio De Janeiro, Brazil, 1986-1998. Mem Inst Oswaldo Cruz. 1999;94:297-304.

4. Pan American Health Organization (PAHO)/World Health Organization. PLISA Health Platform for the Americas. Topics, Dengue, Regional. Available from: http://www.paho.org/data/ indexphp/en/mnu-topics/indicadores-dengue-en/dengue-regional-en.html. [Last accessed on 2018 Jun 13]

5. Sarti E, L'Azou M, Mercado M, et al. A comparative study on active and passive epidemiological surveillance for dengue in five countries of Latin America. Int J Infect Dis. 2016;44:44-9.

6. Pavía-Ruz N, Rojas DP, Villanueva S, et al. Seroprevalence of dengue antibodies in three urban settings in Yucatan, Mexico. Am J Trop Med Hyg. 2018;98:1202-8.

7. Amaya-Larios IY, Martínez-Vega RA, Mayer SV, et al. Seroprevalence of neutralizing antibodies against dengue virus in two localities in the state of morelos, Mexico. Am J Trop Med Hyg. 2014;91:1057-65

8. Hernández-Gaytán SI, Díaz-Vásquez FJ, Duran-Arenas LG, López Cervantes M, Rothenberg SJ. 20 years spatial-temporal analysis of dengue fever and hemorrhagic fever in Mexico. Arch Med Res. 2017;48:653-62.

9. Undurraga EA, Betancourt-Cravioto M, Ramos-Castañeda J, et al. Economic and disease burden of dengue in Mexico. PLoS Negl Trop Dis. 2015;9:e0003547. 
10. Raghupathi $W$, Raghupathi $V$. Big data analytics in healthcare: promise and potential. Health Inf Sci Syst. 2014;2:3.

11. Dantés OG, Sesma S, Becerril VM, Knaul FM, Arreola H, Frenk J. Sistema de salud de México. Salud Pública Méx. 2011; 53:S220-32.

12. Australian Government, Australian Institute of Health and Welfare. Metadata Online Registry. National Healthcare Agreement: PI 18-Selected Potentially Preventable Hospitalisations; 2015. Available from: [Last accessed 2017 Dec 2].

13. International Statistical Classification of Diseases and Related Health Problems, 10th Revision; 2010. Available from: http:// www.apps.who.int/classifications/icd10/browse/2010/en\#/.

14. ENSANUT. Encuesta Nacional de Salud y Nutrición, Resultados Nacionales 2012. Síntesis Ejecutiva; 2012. Available from: http://www.ensanut.insp.mx/doctos/ENSANUT2012_Sint_ Ejec-24oct.pdf.

15. Australian Institute of Health and Welfare. National Healthcare Agreement: PI 18-Selected Potentially Preventable Hospitalisations. Canberra: Australian Institute of Health and Welfare; 2017.

16. MySQL. MySQL Workbench 6.3.6. Available from: https://www. mysql.com/.

17. KNIME. KNIME 3.4.2. Available from: https://www.knime.com/

18. The R Foundation. R: a language and Environment for Statistical Computing. Foundation for Statistical Computing. Vienna, Austria; 2013. Available from: http://www.R-project.org/.

19. San Martín JL, Brathwaite O, Zambrano B, et al. The epidemiology of dengue in the americas over the last three decades: a worrisome reality. Am J Trop Med Hyg. 2010;82:128-35.

20. Hurtado-Díaz M, Riojas-Rodríguez H, Rothenberg SJ, GomezDantés H, Cifuentes E. Short communication: impact of climate variability on the incidence of dengue in Mexico. Trop Med Int Health. 2007:12:1327-37.

21. Richardson V, Sánchez-Uribe E, Esparza-Aguilar M, Esteves-Jaramillo A, Suárez-Idueta L. Contribution of Mexico's universal immunization program to the fourth millennium development goal. Rev Panam Salud Publica. 2014;35:248-55.

22. Zubieta-Zavala A, Salinas-Escudero G, Ramírez-Chávez A, et al. Calculation of the average cost per case of dengue fever in Mexico using a micro-costing approach. PLoS Negl Trop Dis. 2016;10:e0004897.

23. Ladner J, Rodrigues M, Davis B, et al. Societal impact of dengue outbreaks: stakeholder perceptions and related implications. A qualitative study in Brazil, 2015. PLoS Negl Trop Dis. 2017; 11:e0005366.

24. Drennan K, Hicks P, Hart G. Impact of pandemic (H1N1) 2009 on Australasian critical care units. Crit Care Resusc. 2010; 12:223-9.

25. Leuenberger D, Hebelamou J, Strahm S, et al. Impact of the ebola epidemic on general and HIV care in macenta, forest guinea, 2014. AIDS. 2015;29:1883-7.
26. Baurin N, Morley D, Ochiai L, Guergova-Kuras M, Afshar M, Coudeville L. Management of Dengue Hospitalizations in Brazil During and Outside Epidemic Periods: insights from Data Mining. ASTMH 2016 Abstract 212; 2016. Available from: https:// Www.astmh.org/ASTMH/media/Documents/ASTMH2016-Annual-Meeting-Abstract-Book.pdf. [Last accessed 2017 Dec 2].

27. Pan American Health Organization/World Health Organization. Dengue: annual Cases Reported of Dengue; 2017. Available from: http://www.paho.org/hq/indexphp?option=com_topics\& view $=$ rdmore\&cid $=6290 \&$ Itemid $=40734$. [Last accessed 2018 Mar 22].

28. Gaxiola-Robles R, Celis A, Serrano-Pinto V, Orozco-Valerio Mde J, Zenteno-Savín T. Mortality trend by dengue in Mexico 1980 to 2009. Rev Invest Clin. 2012;64:444-51.

29. Rowe EK, Leo YS, Wong JG, et al. Challenges in dengue fever in the elderly: atypical presentation and risk of severe dengue and hospital-acquired infection [corrected]. PLoS Negl Trop Dis. 2014;8:e2777

30. Gonzalez-Gonzalez C, Tysinger B, Goldman DP, Wong R. Projecting diabetes prevalence among Mexicans aged 50 years and older: the future elderly model-mexico (FEM-Mexico). BMJ Open. 2017;7:e017330.

31. Lum LC, Syed Omar SF, Sri La Sri Ponnampalavanar S, et al. Cohorting dengue patients improves the quality of care and clinical outcome. PLoS Negl Trop Dis. 2015;9:e0003836.

32. Halstead SB, Lum LC. Assessing the prognosis of dengue-infected patients. F1000 Med Rep. 2009;1:73.

33. de Silva PK, Jayawardena $P$, Jayarajah $U$, et al. Improving clinical outcomes through setting up of a specialised dengue treatment unit. Int J Adv Res (Indore). 2017;5:1152-3.

34. Sridhar S, Luedtke A, Langevin E, et al. Effect of dengue serostatus on dengue vaccine safety and efficacy. N Engl J Med. 2018; 379:327-40.

35. World Health Organization. Dengue Vaccine: WHO Position Paper; 2018. Available from: http://www.apps.who.int/iris/ bitstream/handle/10665/274315/WER9336.pdf?ua=1. [Last accessed on 2018 Sep 17].

36. Shepard DS, Coudeville L, Halasa YA, Zambrano B, Dayan GH. Economic impact of dengue illness in the Americas. Am J Trop Med Hyg. 2011;84:200-7.

37. Muller DA, Depelsenaire AC, Young PR. Clinical and laboratory diagnosis of dengue virus infection. J Infect Dis. 2017; 215:S89-95.

38. Dantés HG, Farfán-Ale JA, Sarti E. Epidemiological trends of dengue disease in Mexico (2000-2011): a systematic literature search and analysis. PLoS Negl Trop Dis. 2014;8:e3158.

39. Palmu AA, Kilpi TM, Rinta-Kokko H, et al. Pneumococcal conjugate vaccine and clinically suspected invasive pneumococcal disease. Pediatrics. 2015;136:e22-7 